\title{
Nongyrotropy in magnetoplasmas: simulation of wave excitation and phase-space diffusion
}

\author{
U. Motschmann ${ }^{1,2}$, H. Kafemann ${ }^{2}$, M. Scholer ${ }^{3}$ \\ ${ }^{1}$ Deutsche Forschungsanstalt für Luft- und Raumfahrt e. V., Rudower Chaussee 5, D-12484 Berlin, Germany \\ ${ }^{2}$ Institut für Geophysik und Meteorologie, Technische Universität Braunschweig, Mendelssohnstrasse 3, D-38106 Braunschweig, Germany \\ ${ }^{3}$ Max-Planck-Institut für Extraterrestrische Physik, D-85740 Garching, Germany
}

Received: 20 May 1996 / Revised: 9 December 1996 / Accepted: 6 January 1997

\begin{abstract}
Nongyrotropic (gyrophase bunched) ion distributions in a magnetoplasma are studied by analytical methods and by two-dimensional hybrid code simulations. Nongyrotropy may not occur in a plasma being simultaneously homogeneous, stationary, and solenoidal in phase space. A detailed study is performed for a homogeneous and stationary plasma with sources and sinks in phase space. The analytical investigation cast in the framework of linearized Maxwell-Vlasov theory yields a coupling of low-frequency left-handed, righthanded, and longitudinal modes. Nongyrotropic ion distributions are unstable; they excite left-handed waves. The growth rate is comparable to that of the ion ring instability. The hybrid code simulation study confirms the expected propagation direction parallel to the background magnetic field. Three diffusion processes are studied: arc lengthening, arc broadening, and arc radius decreasing corresponding to particle energy diffusion. The characteristic diffusion time-scales are found to be of the order of $10^{1}$ wave cycles.
\end{abstract}

\section{Introduction}

Distribution functions in magnetoplasmas have been extensively studied for several years. These investigations were, however, focused on distribution functions of the type $F\left(v_{\|}, v_{\perp}\right)$, where velocities occur both parallel $\left(v_{\|}\right)$and perpendicular $\left(v_{\perp}\right)$ to the background magnetic field. Such distributions are axially symmetric with respect to the magnetic field and are also termed gyrotropic. When this symmetry is broken, the distribution becomes gyrophase dependent or nongyrotropic.

Nongyrotropy always plays a role when the inhomogeneity scale for plasma interaction processes is smaller than the gyration radius of the corresponding charged particle or when the nonstationary scale is shorter than the gyration period. Another situation for the occurence of nongyrotropy occurs when particle sources and sinks are present in phase space.

Nongyrotropic distributions have been found in several processes in extraterrestrial plasma. Important examples are collisionless shock waves and the interaction of the solar wind with comets. At collisionless shocks nongyrotropy has been detected in the electron population as well as in the ion components (Gosling et al.,1982; Thomsen et al., 1985; Anderson et al., 1985; Sckopke et al., 1990; Fuselier et al., 1990). The interaction of the solar wind with cometary ions may also drive nongyrotropy in the cometary ion component (Motschmann and Glassmeier, 1993; Neubauer et al., 1993; Glassmeier and Neubauer, 1993; Coates et al., 1993). Furthermore, nongyrotropy has been found in the magnetosphere (Mauk et al., 1982) and in the plasma environment of the space shuttle (Cairns, 1990).

The fundamental process for the generation of nongyrotropic distributions is similar in all the afore mentioned situations. Burst-like groups of charged particles move with finite velocity perpendicular to the magnetic field. The particles are picked up as they gyrate around the magnetic field. If the spatial extension of the particle group is smaller than the circumference of the gyration orbit, a nongyrotropic distribution is generated. The required relative velocity may be caused by different mechanisms. At collisionless shocks this velocity results from specular reflection of the particles. The reflected particles run in the opposite direction to the incoming plasma, and are deflected by the perpendicular component of the magnetic field, thereby forming a nongyrotropic distribution in the upstream region. For heavy ions another mechanism exists to form nongyrotropy at shocks. At multi-ion shocks (Motschmann et al., 1991) the velocity loss for heavy ions is smaller than for the protons, which constitute the major component. Thus downstream the heavy ions are faster than the protons and also the magnetic field, as the magnetic field is mainly frozen in the major proton component. The heavy ions are picked up by the magnetic field and may form a nongyrotropic distribution. In the interaction 
between the solar wind and plasma emitted from a comet, the cometary ions just have the solar wind velocity when they are ionized. A perpendicular magnetic field picks up the ions and directs them to a gyration orbit. If this process is restricted to small regions or to short time-scales, as for comet $\mathrm{P} / \mathrm{Grigg}$ Skjellerup (Motschmann and Glassmeier, 1993), a ring distribution is filled up only incompletely, yielding a nongyrotropic distribution.

The first theoretical studies of nongyrotropic distribution functions were carried out in the framework of linear dispersion theory (Sudan, 1965; Fredricks, 1975; Brinca et al., 1992; Motschmann and Glassmeier, 1993). This analysis provides us, besides the growth rate of instability, another remarkable result: nongyrotropy couples modes which are decoupled in the corresponding gyrotropic medium. Brinca et al. $(1992,1993)$ went more deeply into this coupling process by carrying out a particle simulation study for nongyrotorpic electrons.

So far the studies of nongyrotropy have started from homogeneous but nonstationary background plasma where in phase space the ensemble of gyrophase bunched particles gyrates around the magnetic field. In the present paper we compare this nonstationary background state with other studies. In Sect. 2 it is discussed that nongyrotropy is also possible for stationary but inhomogeneous plasmas. Furthermore, nongyrotropy may be maintained even for homogeneous and stationary background with particle sources and sinks in phase space. A study of the mixed case including all conditions simultaneously is given by Cao et al. (1995).

In Sect. 3 we study linear dispersion of nongyrotropic ions which are embedded in a gyrotropic electronproton background, and discuss their stability. We begin with a nongyrotropic fundamental state which is not rotating in phase space but maintained by a steady injection and relaxation of ions. This zero-order state is different from the rotating nongyrotropy studies by Sudan (1965), Brinca et al. (1992), and Motschmann and Glassmeier (1993). We show that for the conditions discussed in our study the stability characteristics of nongyrotropic distributions are not fundamentally different from the gyrotropic ones. The degree of the nongyrotropy-driven coupling of the fundamental modes (L-, R-, P-mode) is strongly determined by the density ratio of the nongyrotropic component compared to the background plasma density. Coupling is stronger at higher nongyrotropic densities.

In Sect. 4 the evolution of nongyrotropy influenced by quasi-linear diffusion processes is studied (Kafemann and Motschmann, 1995). To do this, a spatial twodimensional hybrid code simulation is used. (Scholer et al., 1993). The simulation is initialized with a symmetric nongyrotropic ion distribution in a gyrotropic proton background. Symmetry is used to suppress any zero-order currents in the system. The rotating nongyrotropy is not identical with the situation discussed in Sect. 3. However, the intention of the simulation is to study the diffusion of the nongyrotropic distribution and the saturation of the instability. Imaginable characteristic diffusion processes are arc lengthening (gyrophase diffusion), broadening of the arc, and energy diffusion. All scales were found to be in the order of $10^{1}$ wave cycles. The diffusion is accompanied by the saturation of the instability. The saturation level is rather low $\left(\left|\delta B / B_{0}\right|^{2} \ll 1\right)$. This is because the diffusion increases the plasma beta and the growth rate is strongly decreased.

\section{Unperturbed nongyrotropic states}

We assume a frame in which the unperturbed background magnetic field is directed along $z: \boldsymbol{B}_{0}=\left(0,0, B_{0}\right)$. Wave propagation is studied only parallel to $\boldsymbol{B}_{0}$. Thus the wave vector reads $\boldsymbol{k}=(0,0, k)$. In Sect. 4 the twodimensional simulations will confirm that this restriction is justified. Thus a particle gyrates in the $x-y$-plane. A particle bulk velocity parallel to $\boldsymbol{B}_{0}$ is suppressed.

The Vlasov equation of the undisturbed nongyrotropic plasma component $F_{0}$ extended by sources and sinks then becomes

$\left(\partial_{t}+\boldsymbol{v} \partial_{\boldsymbol{x}}+\frac{q_{0}}{m_{0}}\left[\boldsymbol{v} \times \boldsymbol{B}_{0}\right] \partial_{\boldsymbol{v}}\right) F_{0}=q(\boldsymbol{x}, \boldsymbol{v}, t)-l(\boldsymbol{x}, \boldsymbol{v}, t)$.

The source term we write in the form

$q(\boldsymbol{x}, \boldsymbol{v}, t)=q_{r}(\boldsymbol{x}, t) q_{v}\left(v_{\|}, v_{\perp}, \phi\right)$.

For the loss term we assume the simple case of

$l(\boldsymbol{x}, \boldsymbol{v}, t)=\frac{F_{0}}{\tau}$,

where $\tau$ is a characteristic relaxation time for the nongyrotropic particles. With $\Omega_{0}=q_{0} B_{0} / m_{0}\left(q_{0}\right.$ : charge, $m_{0}$ : mass of the nongyrotropic particle), from Eq. (1) it follows that

$\left(\partial_{t}+\boldsymbol{v} \partial_{\boldsymbol{x}}-\Omega_{0} \partial_{\phi}\right) F_{0}=q_{r}(\boldsymbol{x}, t) q_{v}\left(v_{\|}, v_{\perp}, \phi\right)-\frac{F_{0}}{\tau}$.

This equation can be solved by standard methods; neglecting the loss term it was carried out by Neubauer et al. (1993); the solution of the full equation is given by Cao et al. (1995). Both papers treat the case of nongyrotropy at a weakly active comet where Eq. (4) appears most suitable to describe the distribution of outgassed cometary ions.

Equation (4) contains the impossibility of a nongyrotropic unperturbed state which is simultaneoulsy stationary, homogeneous, and source or sink free. Now we discuss the cases which may be realized in a plasma in separate manner.

Case 1 Homogeneous and stationary nongyrotropic unperturbed state with source and sink in phase space From Eq. (4) we get

$\partial_{\phi} F_{0}-\frac{F_{0}}{\Omega_{0} \tau}=-\frac{q_{r} q_{v}}{\Omega_{0}} ;$

$q_{v}$ is separated into $q_{v}=q_{g}\left(v_{\|}, v_{\perp}\right) q_{\phi}(\phi)$. The solution of Eq. (5) may be written in the form 
$F_{0}=-\frac{q_{r} q_{g}}{\Omega_{0}} \int^{\phi} q_{\phi}\left(\phi^{\prime}\right) \exp \left(\frac{\phi-\phi^{\prime}}{\Omega_{0} \tau}\right) d \phi^{\prime}$.

Without loss of generality we choose the entry point of the injected ions at $\phi=0$, for which $q_{\phi}=\delta(\phi)$. Thus Eq. (6) yields

$F_{0}=-\frac{q_{r} q_{g}}{\Omega_{0}} \exp \left(\frac{\phi}{\Delta}\right), \quad \phi \leq 0$.

The quantity $\Delta=\Omega_{0} \tau$ is the characteristic arc length. The distribution is sketched in Fig. 1a.

Case 2 Nonstationary, homogeneous, and source/sink free nongryotropic unperturbed state

For this case Eq. (4) becomes

$\left(\partial_{t}-\Omega_{0} \partial_{\phi}\right) F_{0}=0$,

with solution

$F_{0}=F_{0}\left(\phi+\Omega_{0} t\right)$.

This describes the gyration of the nongyrotropic particle ensemble with frequency $\Omega_{0}$. This distribution turns up after a temporary particle injection is finished. Its behavior is sketched in Fig. 1b.

Case 3 Inhomogeneous, stationary, and source/sink free nongyrotropic unperturbed state

For this case Eq. (4) may be written as

$\left(\boldsymbol{v} \partial_{x}-\Omega_{0} \partial_{\phi}\right) F_{0}=0$.

Solving this we derive

$F_{0}=F_{0}\left(x+\frac{v_{\perp}}{\Omega_{0}} \sin \phi, \quad y-\frac{v_{\perp}}{\Omega_{0}} \cos \phi, \quad z+\frac{v_{\|}}{\Omega_{0}} \phi\right)$.

This describes the spatial gyration of the nongyrotropic particle ensemble with curvature radius $v_{\perp} / \Omega_{0}$ depending on position. A simple example with a spatial gradient of the magnetic field along $x$ is sketched in Fig. 1c. For $x<0$ the magnetic field vanishes and the particles move straight on. At $x=0$ the particles enter the region of finite magnetic field, where they are forced to gyrate. The particles are turned back and leave the magnetic field region. Within the layer where the particles are turned back a nongyrotropic distribution is formed. The layer-intergrated distribution is sketched in the lower part of Fig. 1c.

The nongyrotropic distribution functions shown in these three cases, as well as combinations of them, satisfy the unperturbed Vlasov equation. In general they carry a current. This situation would not allow a simple unperturbed state as a solution of Maxwell's equations. The current is

$$
\begin{aligned}
& j_{0 x}=q_{0} \int v_{x} F_{0} d^{3} v \propto \int \cos \phi F_{0}\left(v_{\|}, v_{\perp}, \phi\right) d \phi, \\
& j_{0 y}=q_{0} \int v_{y} F_{0} d^{3} v \propto \int \sin \phi F_{0}\left(v_{\|}, v_{\perp}, \phi\right) d \phi .
\end{aligned}
$$

The right-hand terms vanish only when the distribution is symmetric with respect to $\phi$ : i.e., of the form
Nongyrotropy in an open phase space

Nongyrotropy in a nonstationary plasma

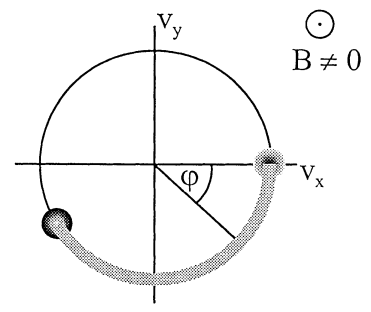

a $\quad \mathrm{F}_{0}(\varphi)$
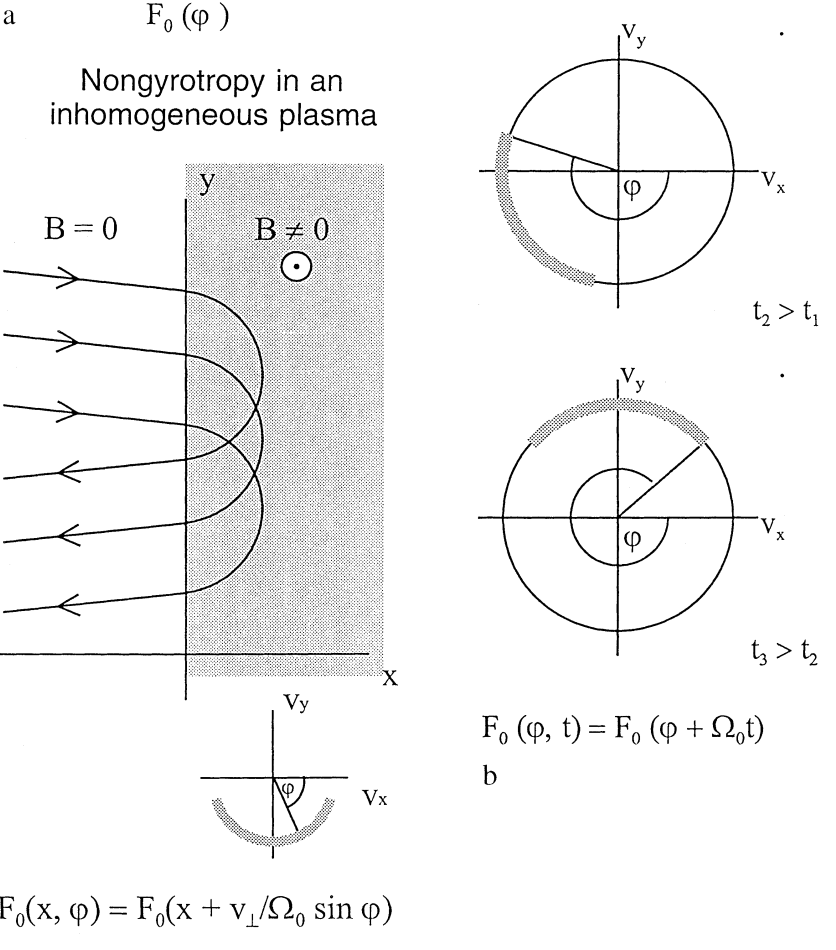

$F_{0}(\varphi, t)=F_{0}\left(\varphi+\Omega_{0} t\right)$

b
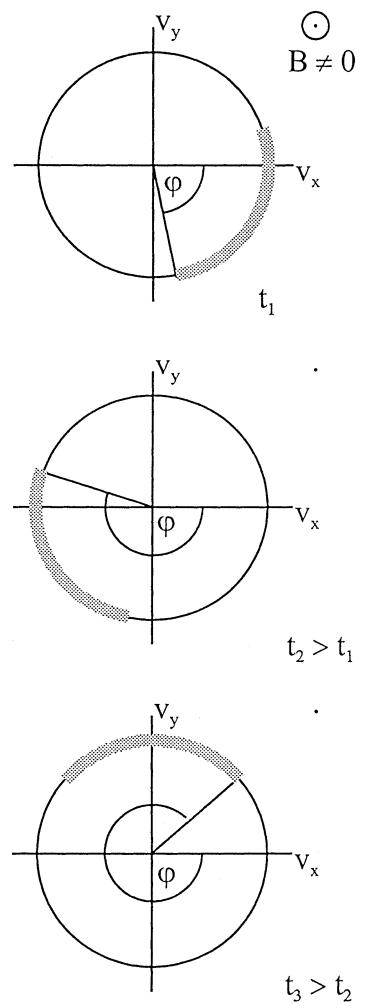

c

Fig. 1. a Nongyrotropic distribution with an ion source and an ion sink in a phase space. b Nonstationary nongyrotropic distribution in phase space; the ion ensemble gyrating in the $v_{x}-v_{y}$ plane sketched at three time-spots $t_{1}<t_{2}<t_{3}$. c Inhomogeneous nongyrotropic distribution; the upper part represents a spatial inhomogeneity by a magnetic field jump; the lower par shows the corresponding nongyrotropy in phase space

$F_{0}(\phi)=F_{0}(\phi \pm \pi)$

An example of such a phase-space symmetry is sketched in Fig. 2. For our fundamental theoretical studies we suppose this symmetry; in real applications, however, this symmetry is hardly ever found. For such unsymmetric situations the restriction to low densities of the nongyrotropic species with respect to the background density keeps the current small so that it may be neglected.

\section{Dispersion theory for waves in nongyrotropic plasmas}

We now study the propagation of waves parallel to the background magnetic field in a nongyrotropic plasma in the frame work of linear Maxwell-Vlasov theory. The 


\section{Symmetric nongyrotropy}

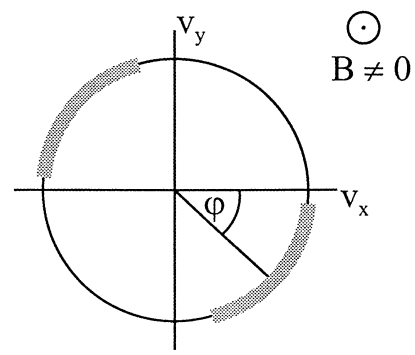

$\mathrm{F}_{0}(\varphi \pm n \pi)$

Fig. 2. Symmetric nongyrotropic distribution; because of symmetry, the unperturbed perpendicular current $j_{\perp}$ vanishes

unperturbed magnetic field is directed along the $z$-axis, $\boldsymbol{B}_{0}=\left(0,0, B_{0}\right)$, as before. The unpeturbed state consists of a gyrotropic background electron-proton plasma with arbitrary but stable distribution functions

$F_{b}=F_{b}\left(v_{\|}, v_{\perp}\right), \quad b=e, p$,

and a nongyrotropic abundance of ions with a distribution function of the form

$F_{0}\left(v_{\|}, v_{\perp}, \phi\right)=F_{g}\left(v_{\|}, v_{\perp}\right) \Phi(\phi)$.

Indices $e, p$, and 0 stand for the electrons, protons, and the nongyrotropic abundance, respectively. Index $b$ is used to mark the entire background. For the nongyrotropic component Case 1 of Section 2 is supposed; the unperturbed distribution is homogeneous and stationary, and the nongyrotropy is maintained by a source and a sink in the phase space.

$\Phi(\phi)$ is $2 \pi$ periodic and may be expanded in to a Fourier series

$\Phi(\phi)=\sum_{m=-\infty}^{\infty} \Phi^{(m)} \exp (-i m \phi)$,

with normalization

$\int \Phi(\phi) d \phi=1$.

The Fourier coefficients are determined via the following integral

$\Phi^{(m)}=\frac{1}{2 \pi} \int \Phi(\phi) \exp (i m \phi) d \phi$.

Let us briefly discuss two extreme situations. In case of gyrotropy the Fourier coefficients become

$\Phi^{(0)}=\frac{1}{2 \pi} ; \quad \Phi^{(m)}=0$ for all $m \neq 0$,

whereas in case of extreme nongyrotropy expressed by

$\Phi(\phi)=\delta(\phi)$

the Fourier coefficients become

$\Phi^{(m)}=\frac{1}{2 \pi}$ for all $m$.
Later we will see that for wave propagation parallel to $\boldsymbol{B}_{0}$ the dispersion relation is only influenced by $\Phi^{(0)}, \Phi^{( \pm 1)}$, and $\Phi^{( \pm 2)}$.

To handle the Maxwell-Vlasov system the $\phi$-independent parts of the distribution functions are taken as Maxwellian of the form

$$
\begin{aligned}
& F_{b}\left(v_{\|}\right)=\frac{1}{\sqrt{\pi} v_{b_{\|}}} \exp \left(-\frac{v_{\|}^{2}}{v_{b_{\|}}^{2}}\right), \quad b=e, p ; \\
& F_{g}\left(v_{\|}\right)=\frac{1}{\sqrt{\pi} v_{0 \|}} \exp \left(-\frac{v_{\|}^{2}}{v_{0_{\|}}^{2}}\right) ;
\end{aligned}
$$

$v_{b \|}$ and $v_{0 \|}$ are parallel thermal velocities. The perpendicular terms $F_{b}\left(v_{\perp}\right)$ and $F_{g}\left(v_{\perp}\right)$ contribute only through their first and second moments. The moments of order $n$ are defined by

$$
\begin{aligned}
& \left\langle v_{b \perp}^{n}\right\rangle=\int_{0}^{\infty}\left(v_{\perp}\right)^{n} F_{b}\left(v_{\perp}\right) v_{\perp} d v_{\perp}, \quad b=e, p ; \\
& \left\langle v_{0 \perp}^{n}\right\rangle=\int_{0}^{\infty}\left(v_{\perp}\right)^{n} F_{g}\left(v_{\perp}\right) v_{\perp} d v_{\perp}, \quad n=1,2 .
\end{aligned}
$$

Then the dispersion relation has the form

$$
\operatorname{det}\left(\begin{array}{lll}
d_{++} & d_{+\|} & d_{+-} \\
d_{\|+} & d_{\|\|} & d_{\|-} \\
d_{-+} & d_{-\|} & d_{--}
\end{array}\right)=0,
$$

with

$$
\begin{aligned}
d_{++}=1 & -\frac{k^{2} c^{2}}{\omega^{2}} \\
& -\sum_{b} \frac{\omega_{b}^{2}}{\omega^{2}}\left[1-\frac{\left\langle v_{b \perp}^{2}\right\rangle}{v_{b \|}^{2}}-\left(\frac{\Omega_{b}}{k v_{b \|}}+\frac{\left\langle v_{b \perp}^{2}\right\rangle}{v_{b \|}^{2}} \zeta_{b}^{+}\right) Z\left(\zeta_{b}^{+}\right)\right] \\
& -2 \pi \Phi^{(0)} \frac{\omega_{0}^{2}}{\omega^{2}}\left[1-\frac{\left\langle v_{0 \perp}^{2}\right\rangle}{v_{0 \|}^{2}}\left(\frac{\Omega_{0}}{k v_{0 \|}}+\frac{\left\langle v_{0 \perp}^{2}\right\rangle}{v_{0 \|}^{2}} \zeta_{0}^{+}\right) Z\left(\zeta_{0}^{+}\right)\right],
\end{aligned}
$$

$d_{+\|}=2 \pi \Phi^{(1)} \frac{\omega_{0}^{2}}{\omega} \frac{\left\langle v_{0 \perp}\right\rangle}{k v_{0 \|}^{2}}\left[1+\zeta_{0}^{+} Z\left(\zeta_{0}^{+}\right)\right]$

$d_{+-}=2 \pi \Phi^{(2)} \frac{\omega_{0}^{2}}{\omega^{2}} \frac{\left\langle v_{0 \perp}^{2}\right\rangle}{v_{0 \|}^{2}}\left[1+\zeta_{0}^{+} Z\left(\zeta_{0}^{+}\right)\right]$,

$d_{\|+}=4 \pi \Phi^{(-1)} \frac{\omega_{0}^{2}}{\omega} \frac{\left\langle v_{0 \perp}\right\rangle}{k v_{0 \|}^{2}}\left[1+\zeta_{0}^{\|} Z\left(\zeta_{0}^{\|}\right)\right]$,

$d_{\|\|}=1+2 \sum_{b} \frac{\omega_{b}^{2}}{\omega^{2}} \zeta_{b}^{\|^{2}}\left[1+\zeta_{b}^{\|} Z\left(\zeta_{b}^{\|}\right)\right]$

$$
+4 \pi \Phi^{(0)} \frac{\omega_{0}^{2}}{\omega^{2}} \zeta_{0}^{\|^{2}}\left[1+\zeta_{0}^{\|} Z\left(\zeta_{0}^{\|}\right)\right],
$$

$d_{\|-}=4 \pi \Phi^{(1)} \frac{\omega_{0}^{2}}{\omega} \frac{\left\langle v_{0 \perp}\right\rangle}{k v_{0 \|}^{2}}\left[1+\zeta_{0}^{\|} Z\left(\zeta_{0}^{\|}\right)\right]$, 


$$
\begin{aligned}
& d_{-+}= 2 \pi \Phi^{(-2)} \frac{\omega_{0}^{2}}{\omega^{2}} \frac{\left\langle v_{0 \perp}^{2}\right\rangle}{v_{0 \|}^{2}}\left[1+\zeta_{0}^{-} Z\left(\zeta_{0}^{-}\right)\right], \\
& d_{-\|}=2 \pi \Phi^{(-1)} \frac{\omega_{0}^{2}}{\omega} \frac{\left\langle v_{0 \perp}\right\rangle}{k v_{0 \|}^{2}}\left[1+\zeta_{0}^{-} Z\left(\zeta_{0}^{-}\right)\right], \\
& d_{--=} 1-\frac{k^{2} c^{2}}{\omega^{2}} \\
&-\sum_{b} \frac{\omega_{b}^{2}}{\omega^{2}}\left[1-\frac{\left\langle v_{b \perp}^{2}\right\rangle}{v_{b \|}^{2}}-\left(-\frac{\Omega_{b}}{k v_{b \|}}+\frac{\left\langle v_{b \perp}^{2}\right\rangle}{v_{b \|}^{2}} \zeta_{b}^{-}\right) Z\left(\zeta_{b}^{-}\right)\right] \\
&-2 \pi \Phi^{(0)} \frac{\omega_{0}^{2}}{\omega^{2}}\left[1-\frac{\left\langle v_{0 \perp}^{2}\right\rangle}{v_{0 \|}^{2}}-\left(-\frac{\Omega_{0}}{k v_{0 \|}}+\frac{\left\langle v_{0 \perp}^{2}\right\rangle}{v_{0 \|}^{2}} \zeta_{0}^{-}\right) Z\left(\zeta_{0}^{-}\right)\right],
\end{aligned}
$$

where $Z(\zeta)$ is the plasma dispersion function defined by

$Z(\zeta)=\int_{-\infty}^{\infty} \frac{\exp \left(-x^{2}\right)}{x-\zeta} d x$

and where we have defined the following terms

$$
\begin{aligned}
& \zeta_{b}^{+}=\frac{\omega-\Omega_{b}}{k v_{b \|}}, \quad \zeta_{b}^{-}=\frac{\omega+\Omega_{b}}{k v_{b \|}}, \quad \zeta_{b}^{\|}=\frac{\omega}{k v_{b \|}}, \quad b=e, p ; \\
& \zeta_{0}^{+}=\frac{\omega-\Omega_{0}}{k v_{0 \|}}, \quad \zeta_{0}^{-}=\frac{\omega+\Omega_{0}}{k v_{0 \|}}, \quad \zeta_{0}^{\|}=\frac{\omega}{k v_{0 \|}} .
\end{aligned}
$$

Details of the calculation are given in the Appendix. $\Omega_{p}$ and $\Omega_{e}$ are the gyrofrequencies of the protons and electrons, respectively. Note that $\Omega_{e}<0$. The gyrofrequency $\Omega_{0}$ of the nongyrotropic ion component is positive for positive ions. In the examples presented in this paper we always take $\Omega_{0}>0 ; \omega_{b}$ and $\omega_{0}$ are the plasma frequencies defined by

$\omega_{b}=\sqrt{\frac{e^{2} n_{b}}{\epsilon_{0} m_{b}}}, \quad b=e, \quad p ; \omega_{0}=\sqrt{\frac{q_{0}^{2} n_{0}}{\epsilon_{0} m_{0}}}$

The particle densities fullfil the condition

$n_{e}=n_{p}+q_{0} n_{0}$

as the plasma is neutral. The terms $A_{b}$ and $A_{0}$ defined by

$A_{b}=\frac{\left\langle v_{b \perp}^{2}\right\rangle}{v_{b \|}^{2}}-1, \quad b=e, p ; \quad A_{0}=\frac{\left\langle v_{0 \perp}^{2}\right\rangle}{v_{0 \|}^{2}}-1$

are the anisotropies of the corresponding components. We set $A_{b}=0(b=e, p)$ throughout the paper to avoid any anisotropy instability of the electron-proton background. For the nongyrotropic component, however, $\left\langle v_{0 \perp}\right\rangle \gg v_{0 \|}$ and $\left\langle v_{0 \perp}^{2}\right\rangle \gg v_{0 \perp}^{2}\left(A_{0} \gg 1\right)$ are supposed, as $\left\langle v_{0 \perp}\right\rangle$ and $\left\langle v_{0 \perp}^{2}\right\rangle$, respectively, come out of the radius of an incomplete ring in phase space. Of course, the radius of the ring is much bigger than its spreading, which is of the same order as $v_{0 \perp}$. In our discussion we regard $\alpha$ particles as the nongyrotropic ion component for which $\Omega_{0}=\Omega_{p} / 2$; We normalize all frequencies to $\Omega p$ and all velocities to the Alfvén velocity $v_{A}$ given by
$v_{A}=\frac{B_{0}}{\sqrt{\mu_{0}\left(m_{p} n_{p}+m_{0} n_{0}\right)}}$.

We later introduce the parallel components of the plasma betas which have the form

$\beta_{b \|}=\frac{\left\langle v_{b \|}^{2}\right\rangle}{v_{A}^{2}}, \quad b=e, p ; \quad \beta_{0 \|}=\frac{\left\langle v_{0 \|}^{2}\right\rangle}{v_{A}^{2}}$.

Now we discuss the dispersion relation Eq. (24). First one sees that the off-diagonal elements of Eq. (24) are immediately connected with the nongyotropy. This is valid for any form of nongyrotropy, and was found already in the first paper on this topic (Sudan, 1965). For a gyrotropic plasma $\Phi^{( \pm 1)}=\Phi^{( \pm 2)}=0$, and Eq. (24) splits into the three independent equations

$d_{++}=d_{\|\|}=d_{--}=0$.

Then the nongyrotropy approaches a full ring and the three equations of Eq. (27) describe the three fundamental $L$-, $R$-, and $P$-modes. As we are interested in the consequences of nongyrotropic ion abundance only the ion modes are discussed. No attention is payed to highfrequency electron modes, as they are not really changed.

The dispersion relation for the $L$-mode is $d_{++}=0$. It is the left-hand polarized mode which approaches the Alfvén mode when $\omega \rightarrow 0$. The L-mode is unstable because of the ring instability. As is to be seen from Eq. (24a), the shape of $F_{0}\left(v_{\perp}\right)$ is irrelevant and only the value $\left\langle v_{0 \perp}^{2}\right\rangle$ matters; in general, this instability is an anisotropy instability. The ring instability is a special case. The dispersion and growth rate are shown in Fig. $3 \mathrm{a}$. The number density $n_{0}$ of the nongyrotropic component is 2 percent of the background protons $\left(n_{0}=0.02 n_{p}\right)$. The other parameters are $A_{0}=400$, $A_{p}=A_{e}=0$, and $\beta_{0 \|}=\beta_{p \|}=\beta_{e \|}=0.01$. The unstable branch is that which is referenced to the ring component. The maximum growth rate excites frequencies just below the gyrofrequency of the ring ions.

The R-mode is represented by the expression $d_{--}=0$. It describes the right-hand polarized mode which approaches the fast mode when $\omega \rightarrow 0$ and the whistler mode when $\Omega_{e} \gg \omega \gg \Omega_{p}$. The dispersion is shown in Fig. $3 \mathrm{~b}$ showing that the R-mode is completely stable. The parameters are the same as for Fig. 3a. As the ring ions are positively charged the R-mode has no resonances in the ion frequency region.

P-mode: $d_{\|\|}=0$ describes the longitudinal mode. It corresponds to the ion sound or slow mode, and only occurs for extremely low frequencies.

Mode coupling: one major point of nongyrotropy is that the off-diagonal elements of Eq. (24) become finite. Thus the three fundamental modes described are no longer independent but are coupled. To keep this coupling transparent we concentrate our discussion to the coupling of the L- and R-modes only. This is achieved by a symmetric nongyrotropy as shown in Fig. 2. For this $\Phi^{( \pm 2)} \neq 0$, whereas $\Phi^{( \pm 1)}=0$ and thus the P-mode is still decoupled from the others. We assume a strong nongyrotropy by taking $\Phi^{(+2)}=\Phi^{(-2)}=1 /(2 \pi)$. For the same 

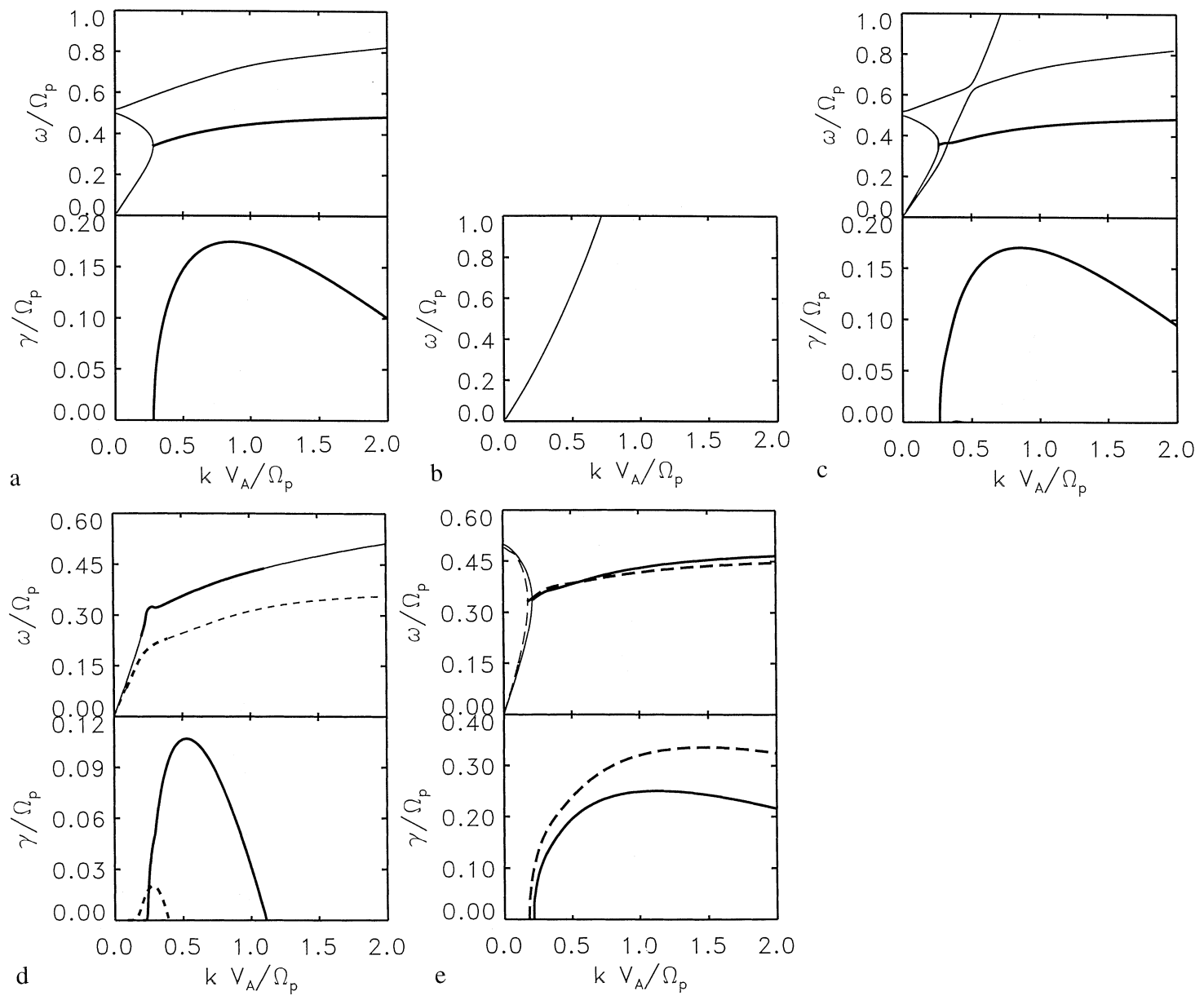

Fig. 3. a Dispersion (upper panel) and growth rate (lower panel) of the L-mode in gyrotropic plasma; the fat line is the unstable branch. b Dispersion of the R-mode in gyrotropic plasma; this mode is completely stable. c Dispersion (upper panel) and growth rate (lower panel) of coupled L-R modes in nongyrotropic plasma; the fat line is the unstable branch. d Dispersion (upper panel) and growth rate (lower panel) of coupled L-R modes in a nongyrotropic plasma at higher

parameters used in Fig. 3a and $b$ we now obtain the behavior as shown in Fig. 3c. The L- and R-modes are recognized. The cross points caused by their superposition show some different behavior. The upper cross point at about $\omega / \Omega_{p} \approx 0.65, k v_{A} / \Omega_{p} \approx 0.5$ splits. Both original branches were stable and remain so as a result of the coupling. Another cross point at about $\omega / \Omega_{p} \approx 0.36$, $k v_{A} / \Omega_{p} \approx 0.33$ does not split. We guess that this is a singular touch point of two dispersion surfaces spanned over the $k_{\|}-k_{\perp}$ plane. Any deviation from the parallel propagation direction should result in a splitting. Analogous situations in multicomponent plasmas are already known (André, 1984). The influence of the coupling to the instability is of minor importance. The unstable branch of the L-mode is only weakly changed.

The influence of higher plasma beta and higher abundance of the nongyrotropic species is shown in the next two figures. Fig. 3d depicts the unstable branch when the betas are increased by one and two orders of magnitude being then $\beta_{0 \|}=\beta_{p \|}=\beta_{e \|}=0.1$ and 1.0, respectively. The other parameters are the same as in Fig. 3c. Especially for $\beta_{0 \|}=\beta_{p \|}=\beta_{e \|}=1.0$ the growth betas; the solid lines represent $\beta_{0 \|}=0.1$; the dashed lines represent $\beta_{0 \|}=1.0$; the fat lines mark the unstable regions; only branches with instability are drawn. e Dispersion (upper panel) and growth rate (lower panel) of coupled L-R modes at higher abundance of the nongyrotropic species; the solid lines represent $n_{0}=0.05 n_{p}$; the dashed lines represent $n_{0}=0.1 n_{p}$; the fat lines mark the unstable regions; completely stable branches are suppressed

rate goes down drastically and nearly no real wave growth is to be expected. An increase in the growth rate, however, is provided at higher abundance of particles of the nongyrotropic component as shown in Fig. 3e. $\alpha$ particle densities are $n_{0}=0.05 n_{p}$ and $n_{0}=0.1 n_{p}$, whereas the other parameters are the same as in Fig. 3c. The maximum growth rate goes up from $\gamma_{\max } \approx 0.17 \Omega_{p}^{-1}$ at $n_{0}=0.02 n_{p}$. to $\gamma_{\max } \approx 0.35 \Omega_{p}^{-1}$ at $n_{0}=0.1 n_{p}$. The dispersion relation Eq. (24) based on the stationary and homogeneous zero-order nongyrotopic distribution function Eq. (7) is different from the dispersion relation based on the gyrating zero-order distribution function Eq. (9). The implications with respect to weakly outgassing comets are discussed by Cao et al. (1995).

\section{Hybrid simulation of phase-space diffusion and saturation of the instability}

Within the framework of linear dispersion theory only the initial stages of wave growth may be described. To go further and to include diffusion and saturation we sim- 
ulate the wave excitation with a hybrid code (Scholer et al., 1993). The simulation method is $2 \frac{1}{2} 2^{-d i m e n s i o n a l, ~}$ which allows for the possibility to include wave propagation directions arbitrarily orientated relative to the background magnetic field. However, we will find that waves are excited only parallel to the background magnetic field, strongly confirming our assumption Sect. 3. In the code all ions (nongyrotropic $\alpha$-particles and gyrotropic background protons) are treated as individual particles, thereby corresponding to a complete kinetic description for the ion behavior. The electrons are a massless fluid background. At $t=0$ the simulation is started with a nongyrotropic distribution of $\alpha$-particles as shown in Fig. 4. We choose a symmetric distribution to suppress any zero-order currents. The $\alpha$ particles are embedded in a gyrotropic proton-electron background. The situation corresponds to Case 2 of Sect. 2. For the particle simulation we prefer this case as it is much easier to handle than the open phase space discussed in Case 1.

At the beginning of the simulation run a broad spectral range of waves is excited. This switch-on phase is shown in Fig. 5a. The R-mode with its mainly linear $\omega-k$ dependence is seen, as well as the L-mode with its resonance below $\omega / \Omega_{p} \approx 0.5$. At this stage the wave excitation is dominant by virtue of the switch-on perturbation. Later on in the simulation the wave excitation develops into that shown in Fig. 5b. The instability is now the dominant mechanism. Only the unstable L-mode survives for wave numbers near the maximum growth rate.

The good agreement is somewhat surprising as the dispersion branches are provided from the open phase space model (Case 1), whereas the simulation runs in a closed phase space (Case 2). Apparently at the early stage (see Fig. 5a) of the simulation where diffusion and saturation are not yet developed, it is of minor importance by which process nongyrotropy is maintained. At a later stage (see Fig. 5b) the agreement is less essential,

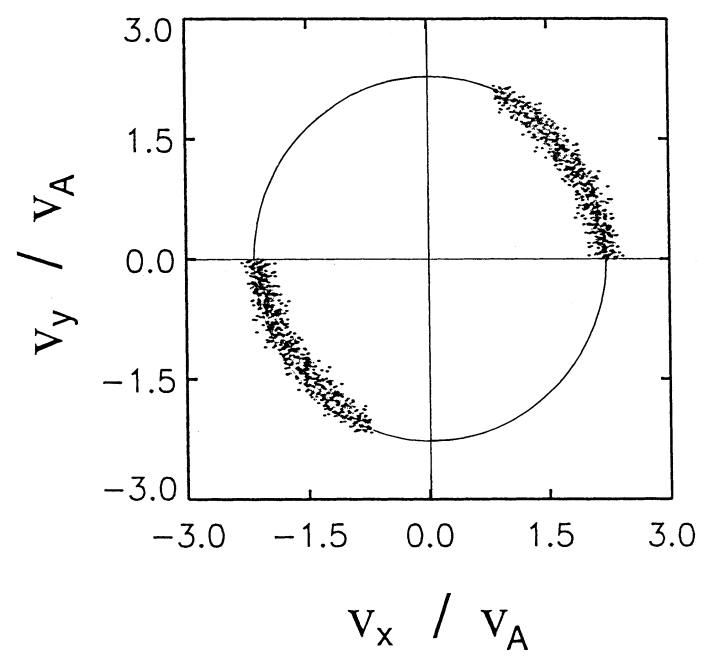

Fig. 4. Sketch of the nongyrotropic initial distribution
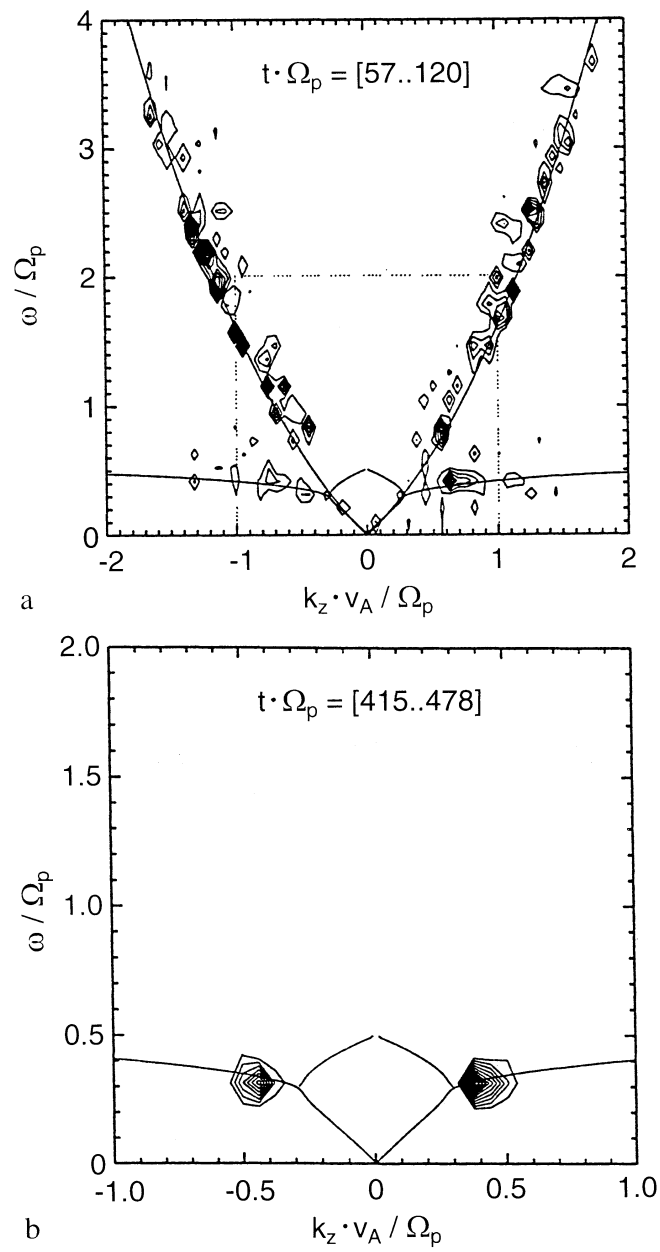

Fig. 5. a $\omega-k_{z}$ diagram at an early simulation time; the solid lines correspond to dispersion from linear dispersion theory; b $\omega-k_{z}$ diagram at an advanced simulation time; only the unstable mode with maximum growth rate survives

as a maximum growth rate just below the ion gyrofrequency is predicted by dispersion theory in an open as well as in a closed phase space.

Now we will study the phase-space diffusion of the nongyrotropic species. In Fig. 6 the phase space with the possible diffusion processes is sketched. Three processes are distinguished. The diffusion of the mean perpendicular velocity $\left\langle v_{\perp}\right\rangle$, the arc spreading $\sigma\left(v_{\perp}\right)=$ $\sqrt{\left\langle\left(v_{\perp}-\left\langle v_{\perp}\right\rangle\right)^{2}\right\rangle}$ and the increasing of the arc length $\Delta=\left\langle v_{\perp}\right\rangle \phi$. We made several runs with different parameters. As a typical result we will set off a reference run where the density of the nongyrotropic particles is 2 percent of the proton background $\left(n_{0}=0.02 n_{p}\right)$, the mean ring velocity $\left\langle v_{0 \perp}\right\rangle=2 v_{A}$, the initial beta $\beta_{0 \|}=0.01$ giving an initial nongyrotropic anisotropy of $A_{0}=400$. Initial arc length is $\phi=2 \times \pi / 4$ and arc spreading is $\sigma=0.01 v_{A}$. The electron-proton background is gyrotropic with a thermal initialization of $\beta_{p\|\|}=\beta_{e \|}=\beta_{0 \|}$. The simulation results are depicted in Fig.7.

The sequence of phase-space plots in Fig.7a provides us with a qualitative impression of the diffusion of the 


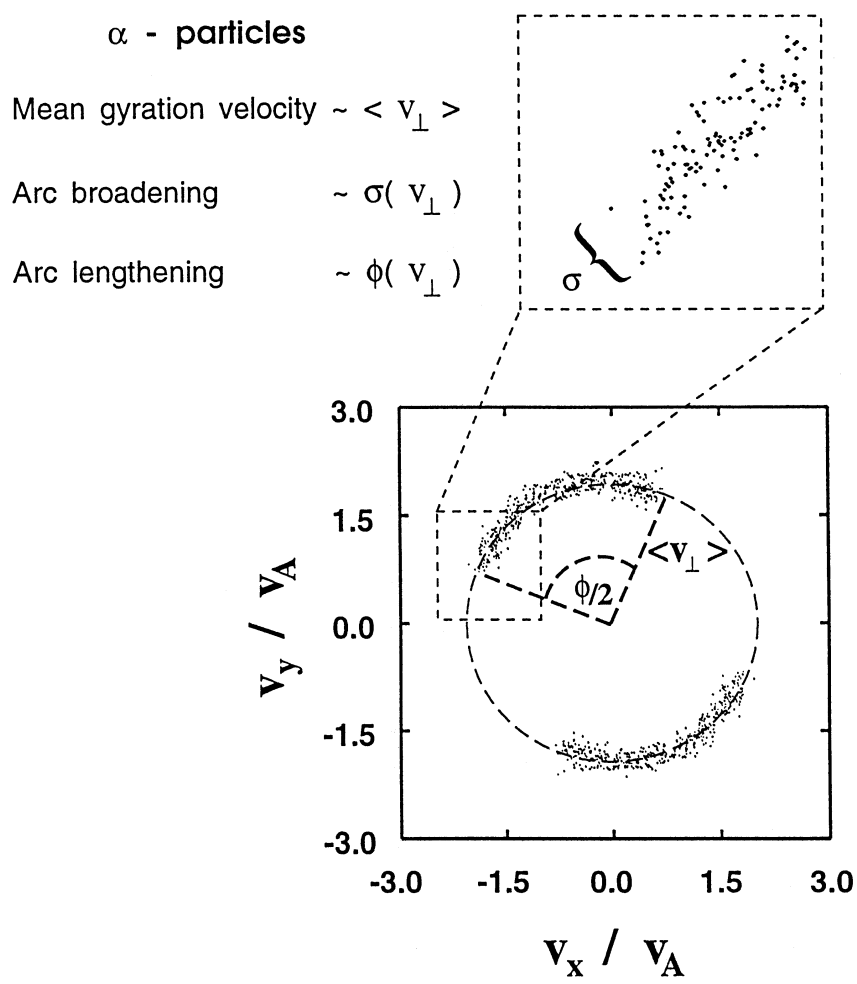

Fig. 6. Sketch of the diffusion processes in phase space

nongyrotropic distribution. At early times $\left(t=12.57 \Omega_{p}^{-1}\right.$, top $)$ the phase space is still in its initial configuration despite of the gyration of the entire distribution. At later times $\left(t=125.66 \Omega_{p}^{-1}\right.$, center $)$ the arcs are broadened and lengthened and the mean velocity is decreased. Finally $\left(t=251.33 \Omega_{p}^{-1}\right.$, bottom $)$ the arcs are nearly grown together.

Diffusion and saturation are shown in more detail in Fig. $7 \mathrm{~b}$. Whereas the mean ring velocity $\left\langle v_{\perp}\right\rangle$ and the anisotropy $A_{0}$ decrease as expected, the thermal velocities go up, depicted by the increase in $\sigma$ and $\beta_{0 \|}$. The growth phase extends up to about $t \approx 1300 \Omega_{p}^{-1}$ and is well distinguished from the saturation phase at $t \geq 130 \Omega_{p}^{-1}$. Saturation in the present context does not mean that final stationary conditions are reached but temporary evolution runs very slowly compared with the early growth phase. The magnetic fluctuations $\left|\delta B / B_{0}\right|^{2}$ show a rather low saturation level. This may be understood as $\beta_{0 \|}$ increasing strongly during the growth phase. However, from linear dispersion theory we have learnt that the growth rate $\gamma$ decreases drastically when $\beta_{0 \|}$ approaches 1, and thus the fluctuation level of $\left|\delta B / B_{0}\right|^{2}$ is kept nearly constant. Thus, the reservoir of free particle energy is exploited only minimally. Obviously the contribution of the waves to the total energy balance is of orders of magnitude below the kinetic particle energy. The contour plot of the spectral density provides us with a mean frequency and a mean wave number at $\omega / \Omega_{p} \approx 0.25$ and $k v_{A} / \Omega_{p} \approx 0.3$. These results are in reasonable agreement with linear dispersion theory as discussed in Sect. 3.

We emphasize that the characteristic time-scales for the three diffusion processes are all in the same order.
The coincidence of these time-scales does not hint at a special arc lengthening process. Arcs are lengthened synchronously to their spreading. We call this process local thermalization, as a group of particles diffuses from its position in phase space. Spreading and lengthening of an arc is of the same absolute value. The spreading appears more obvious but this is only a relative effect. In our reference run we found the diffusion time-scale of $t_{d} \approx 130 \Omega_{p}^{-1}$; this corresponds to about ten gyrations of an $\alpha$-particle.

The influence of the nongyrotropic abundance to this time-scale is shown in Fig. 8. The decrease in the nongyrotropic anisotropy and the increase in the wave fluctuations are depicted for $n_{0}=0.01 n_{p}$ additionally to our reference run with $n_{0}=0.02 n_{p}$. Lower abundance increases the diffusion time-scale more than adequately.

\section{Summary}

For nongyrotropic particle distribution functions in magnetoplasmas the axial symmetry in phase space is broken. Such distributions are gyrophase dependent. Nongyrotropy may occur in the ion as well as in the electron components. In this paper we have mainly studied nongyrotropy in an ion component. An important property is that these distributions may not be simultaneously homogeneous, stationary, and source/ sink-free in phase space. They are found in magnetoplasmas when inhomogeneity scale lengths are smaller than the ion gyroradius, when nonstationary scale lengths are shorter than the gyroperiod, or when there are sources and sinks in phase space of the corresponding plasma species. So far nongyrotropy has been observed particularly in extraterrestrial plasmas. It occurs at burst-like reflections of electrons or ions at collisionless shocks, in heavy ion components of multiion shocks in the downstream region, and at solar windcomet interactions, especially in the case of small comets.

In this paper the characteristics of nongyrotropic ion distributions were studied based on linear MaxwellVlasov dispersion theory as well as with a $2 \frac{1}{2}$-dimensional hybrid code simulation. The two spatial dimensions of the model include propagation directions with arbitrary angle to the background magnetic field. However, it is found that waves propagate only in a parallel direction. Thus the dispersion analysis is restricted to this situation. The consequence is that nongyrotropic distributions have similar dispersion and instability properties as the corresponding gyrotropic ones. Already known for gyrotropic ion distributions the fundamental L-, R-, and P-modes are also found in nongyrotropic case. The unstable L-mode maintains its instability characteristics. We conclude that gyrophase bunching does not influence the growth rate essentially if the corresponding gyrotropic distribution is already unstable. In a stable gyrotropic medium, however, Brinca et al. (1993) discussed that gyrophase organization is of essential importance by destabilization of a formerly stable plasma. Completely new for nongyrot- 

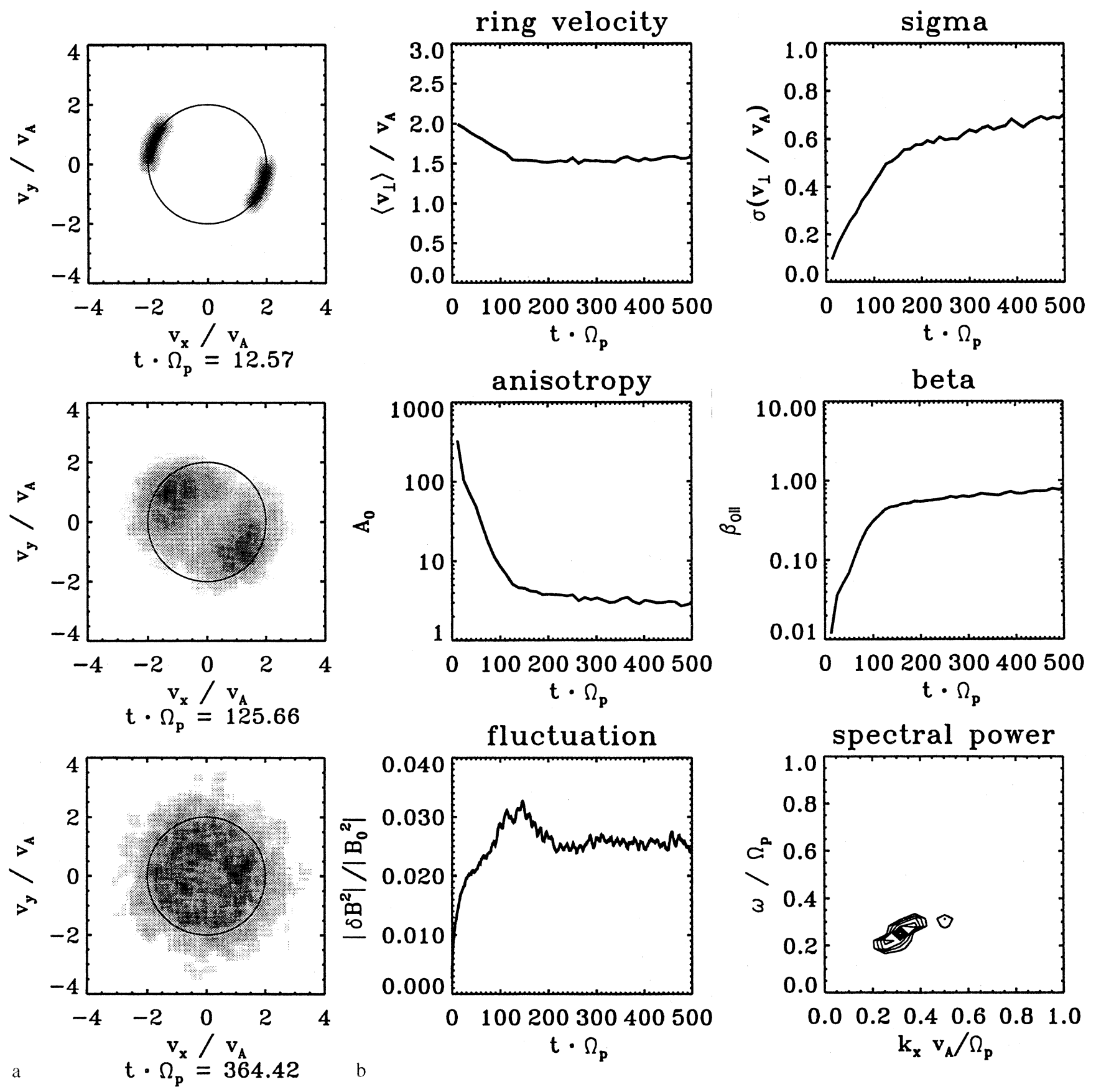

Fig. 7. a Nongyrotropic phase space density for three different simulation times. b Evolution of phase-space parameters provided by the simulation of a nongyrotropic plasma; the panels represent the mean ring velocity, the arc spreading, the anisotropy, the parallel beta,

ropy is the coupling of L-, R-, and P-modes with each other. In this paper a detailed study of L-R coupling is performed. In principle the coupling takes place at any arbitary strength of nongyrotropy and particle density. A remarkable influence, however, is seen for strong nongyrotropy and particle densities at least in the percent region of the background plasma.

The phase-space diffusion of nongyrotropic ions studied by the hybrid code simulation may be imagined to separate in three processes; arc lengthening, arc and the magnetic fluctuations; the panel bottom right represents a contour plot of the magnetic spectral density in the saturation phase $\left(t \Omega_{p}=[125 \ldots 325]\right)$

broadening, and diffusion of the distribution radius. The characteristic diffusion times are all in the same order. In our simulation study performed with a strong initial anisotropy, saturation is reached after a time-scale corresponding to about ten gyrations of the nongyrotropic particles. The wave fluctuations saturate at a rather low level, though most of the free particle energy stored in the nongyrotropic ring is still available. However, during the growth phase the plasma beta is strongly increased, decreasing coincidentally the growth 

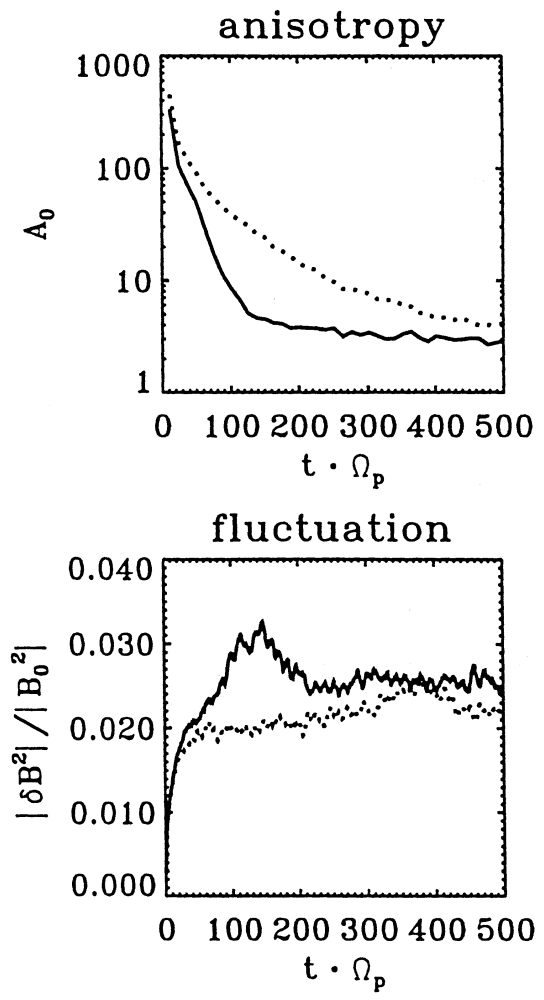

Fig. 8. Evolution of anisotropy and magnetic fluctuation level for two nongyrotropic particle densities; the solid lines represent $n_{0}=0.02 n_{p}$; the dotted lines represent $n_{0}=0.01 n_{p}$.

rate of the instability. Thus the wave fluctuation level remains nearly constant on a low level.

Acknowledgements. The authors are grateful to K. H. Glassmeier and T. I. Woodward for helpful discussions. The work of U. M. is supported by the Deutsche Forschungsgemeinschaft through a Werner-Heisenberg-Stipendium and through the grant Mo 539/5.

Topical Editor K.- H. Glaßmeier thanks A. L. Brinca and C. Mazelle for their help in evaluating this paper.

\section{Appendix}

The Vlasov equation for the linear disturbance $F_{1}$ of the nongyrotropic distribution function is

$\left(\partial_{t}-\Omega_{0} \partial_{\phi}+v_{\|} \partial_{z}\right) F_{1}=-\frac{q_{0}}{m_{0}}(\boldsymbol{E}+\boldsymbol{v} \times \boldsymbol{B}) \partial_{\boldsymbol{v}} F_{0}$.

The gyrophase dependence is expanded in a Fourier series yielding

$F_{1}\left(z, t, v_{\|}, v_{\perp}, \phi\right)=\sum_{n=\infty}^{\infty} F_{1}^{(n)}\left(z, t, v_{\|}, v_{\perp}\right) \exp (-i n \phi)$.

After Fourier transformation of Eq. (A1) with respect to $z$ and $t$ one obtains

$$
\begin{aligned}
& \sum_{n=-\infty}^{\infty}\left[-i\left(\omega-n \Omega_{0}-k v_{\|}\right)\right] F_{1}^{(n)} \exp (-i n \phi) \\
&=-\frac{q_{0}}{m_{0}} \sum_{m=-\infty}^{\infty}\left\{\left[\left(1-\frac{k v_{\|}}{\omega}\right)\left(\partial_{v_{\perp}} F_{g}-\frac{m}{v_{\perp}} F_{g}\right)+\frac{k v_{\perp}}{\omega} \partial_{v_{\|}} F_{g}\right]\right. \\
& \quad \Phi^{(m)} \exp [-i(m+1) \phi] E_{+}(\omega, k) \\
&+\partial_{v_{\|}} F_{g} \Phi^{(m)} \exp [-i m \phi] E_{\|}(\omega, k) \\
&+ {\left[\left(1-\frac{k v_{\|}}{\omega}\right)\left(\partial_{v_{\perp}} F_{g}+\frac{m}{v_{\perp}} F_{g}\right)\right.} \\
&\left.\left.+\frac{k v_{\perp}}{\omega} \partial_{v_{\perp}} F_{g}\right] \Phi^{(m)} \exp [-i(m-1) \phi] E_{-}(\omega, k)\right\} .
\end{aligned}
$$

From this we find

$$
\begin{aligned}
& F_{1}^{(n)}=-i \frac{q_{0}}{m_{0}} \frac{1}{\omega-n \Omega_{0}-k v_{\|}} \\
&\left\{\left[\left(1-\frac{k v_{\|}}{\omega}\right)\left(\partial_{v_{\perp}} F_{g}-\frac{n-1}{v_{\perp}} F_{g}\right)+\frac{k v_{\perp}}{\omega} \partial_{v_{\|}} F_{g}\right]\right. \\
& \Phi^{(n-1)} E_{+}(\omega, k) \\
&+\left[\partial_{v_{\|}} F_{g}\right] \Phi^{(n)} E_{\|}(\omega, k) \\
&+\left[\left(1-\frac{k v_{\|}}{\omega}\right)\left(\partial_{v_{\perp}} F_{g}+\frac{n+1}{v_{\perp}} F_{g}\right)+\frac{k v_{\perp}}{\omega} \partial_{v_{\|}} F_{g}\right] \\
&\left.\Phi^{(n+1)} E_{-}(\omega, k)\right\},
\end{aligned}
$$

where we have introduced the circular polarized field amplitudes

$E_{ \pm}=\frac{1}{2}\left(E_{x} \pm i E_{y}\right) ; \quad E_{\|}=E_{z}$

Analogously we have set

$v_{ \pm}=\frac{1}{2}\left(v_{x} \pm i v_{y}\right)=\frac{v_{\perp}}{2} \exp ( \pm i \phi)$.

The perturbation current, defined by

$\boldsymbol{j}(\omega, k)=q_{0} \int \boldsymbol{v} F_{1}\left(\omega, k, v_{\|}, v_{\perp}, \phi\right) d^{3} v$,

may then also be expressed in terms of the circular polarized amplitudes $j_{+}, j_{\|}$and $j_{-}$yielding

$j_{+}=q_{0} 2 \pi \int \frac{v_{\perp}}{2} F_{1}^{(1)} v_{\perp} d v_{\perp} d v_{\|}$

$j_{\|}=q_{0} 2 \pi \int v_{\|} F_{1}^{(0)} v_{\perp} d v_{\perp} d v_{\|}$,

$j_{-}=q_{0} 2 \pi \int \frac{v_{\perp}}{2} F_{1}^{(-1)} v_{\perp} d v_{\perp} d v_{\|}$.

Putting Eq. (A4) in Eq. (A8) and using

$\boldsymbol{j}=\sigma \boldsymbol{E}$

the conductivity tensor $\sigma$ follows in the form 


$$
\begin{aligned}
& \sigma_{++}=-i \frac{q_{0}^{2}}{m_{0}} 2 \pi \Phi^{(0)} \int \frac{v_{\perp} / 2}{\omega-k v_{\|}-\Omega_{0}} \\
& \times\left[\left(1-\frac{k v_{\|}}{\omega}\right) \partial_{v_{\perp}}+\frac{k v_{\perp}}{\omega} \partial_{v_{\|}}\right] F_{g} v_{\perp} d v_{\perp} d v_{\|}, \\
& \sigma_{+\|}=-i \frac{q_{0}^{2}}{m_{0}} 2 \pi \Phi^{(1)} \int \frac{v_{\perp} / 2}{\omega-k v_{\|}-\Omega_{0}} \partial_{v_{\|}} F_{g} v_{\perp} d_{v_{\perp}}, d v_{\|}, \\
& \sigma_{+-}=-i \frac{q_{0}^{2}}{m_{0}} 2 \pi \Phi^{(2)} \int \frac{v_{\perp} / 2}{\omega-k v_{\|}-\Omega_{0}} \\
& \times\left[\left(1-\frac{k v_{\|}}{\omega}\right)\left(\partial_{v_{\perp}}+\frac{2}{v_{\perp}}\right)+\frac{k v_{\perp}}{\omega} \partial_{v_{\|}}\right] \\
& \times F_{g} v_{\perp} d v_{\perp} d v_{\|} \text {, } \\
& \sigma_{\|+}=-i \frac{q_{0}^{2}}{m_{0}} 2 \pi \Phi^{(-1)} \int \frac{v_{\|}}{\omega-k v_{\|}} \\
& \times\left[\left(1-\frac{k v_{\|}}{\omega}\right)\left(\partial_{v_{\perp}}+\frac{1}{v_{g}}\right)+\frac{k v_{\perp}}{\omega} \partial_{v_{\|}}\right] \\
& \times F_{g} v_{\perp} d v_{\perp} d v_{\|} \text {, } \\
& \sigma_{\|\|}=-i \frac{q_{0}^{2}}{m_{0}} 2 \pi \Phi^{(0)} \int \frac{v_{\|}}{\omega-k v_{\|}} \partial_{v_{\|}} F_{g} v_{\perp} d v_{\perp} d v_{\|}, \\
& \sigma_{\|-}=-i \frac{q_{0}^{2}}{m_{0}} 2 \pi \Phi^{(1)} \int \frac{v_{\|}}{\omega-k v_{\|}} \\
& \times\left[\left(1-\frac{k v_{\|}}{\omega}\right)\left(\partial_{v_{\perp}}+\frac{1}{v_{\perp}}\right)+\frac{k v_{\perp}}{\omega} \partial_{v_{\|}}\right] \\
& \times F_{g} v_{\perp} d v_{\perp} d v_{\|}, \\
& \sigma_{-+}=-i \frac{q_{0}^{2}}{m_{0}} 2 \pi \Phi^{(-2)} \int \frac{v_{\perp} / 2}{\omega-k v_{\|}+\Omega_{0}} \\
& \times\left[\left(1-\frac{k v_{\|}}{\omega}\right)\left(\partial_{v_{\perp}}+\frac{2}{v_{\perp}}\right)+\frac{k v_{\perp}}{\omega} \partial_{v_{\|}}\right] \\
& \times F_{g} v_{\perp} d v_{\perp} d v_{\|} \text {, } \\
& \sigma_{-\|}=-i \frac{q_{0}^{2}}{m_{0}} 2 \pi \Phi^{(-1)} \int \frac{v_{\perp} / 2}{\omega-k v_{\|}+\Omega_{0}} \partial_{v_{\|}} F_{g} v_{\perp} d v_{\perp} d v_{\|}, \\
& \sigma_{--}=-i \frac{q_{0}^{2}}{m_{0}} 2 \pi \Phi^{(0)} \int \frac{v_{\perp} / 2}{\omega-k v_{\|}+\Omega_{0}} \\
& \times\left[\left(1-\frac{k v_{\|}}{\omega}\right) \partial_{v_{\perp}}+\frac{k v_{\perp}}{\omega} \partial_{v_{\|}}\right] \\
& \times F_{g} v_{\perp} d v_{\perp} d v_{\|} \text {. }
\end{aligned}
$$

Setting Eqs. (22) and (23) into Eq. (A10) and appending corresponding terms for the gyrotropic electron-proton background, the perturbation current of the full plasma composition is completely determined. Via Maxwell's equations, the dispersion tensor Eq. (24) follows in a straightforward fashion.

\section{References}

Anderson, K. A., R. P. Lin, C. Gurgiolo, G. K. Parks, D. W. Potter, S. Werden, and $\mathbf{H}$. Reme, A component of nongyrotropic (phase-bunched) electrons upstream from the earth's bow shock, J. Geophys, Res., 90, 10809-10814, 1985.

André M., Dispersion surfaces, Kiruna Geophysical Institute Preprint 067, 1984.

Brinca, A. L., L. Borda de Água, and D. Winske, Nongyrotropy as a source of instability and mode coupling, Geophys. Res. Lett., 12, 2445-2448, 1992.

Brinca, A. L., Y. Omura, and H. Matsumoto, Linear coupling effects originated in electron nongyrotropy, J. Geophys. Res., 98, 21071-21076, 1993.

Cairns, I. H., Transition from ring to beam arc distributions of water ions near the space shuttle orbiter, J. Geophys. Res., 95, 15167-15173, 1990.

Cao, J. B., C. Mazelle, G. Belmont, and H. Reme, The nongyrotropy of heavy newborn ions at comet Grigg-Skjellerup and corresponding instability, J. Geophys. Res., 100, 23379-23388, 1995.

Coates, A. J., A. D. Johnstone, B. Wilken, and F. M. Neubauer, Velocity space diffusion and nongyrotropy of pickup water group ions at comet Grigg-Skjellerup, J. Geophys. Res., 98, 20985-20994, 1993.

Fredricks, R. W., A model for generation of bow-shock-associated upstream waves, J. Geophys. Res., 80, 7-17, 1975.

Fuselier, S. A., O. W. Lennartsson, M. F. Thomsen, and C. T. Russell, Specularly reflected $\mathrm{He}^{2+}$ at high Mach number quasiparallel shocks, J. Geophys. Res., 95, 4319-4325, 1990.

Glassmeier, K. H., and F. M. Neubauer, Low-frequency electromagnetic plasma waves at comet $\mathrm{P} /$ Grigg-Skjellerup: overview and spectral characteristics, J. Geophys. Res., 98, 20921, 1993.

Gosling, J. T., M. F. Thomsen, S. J. Bame, W. C. Feldman, G. Paschmann, and N. Sckopke, Evidence for specularly reflected ions upstream from the quasi-parallel bow shock, Geophys. Res. Lett., 9, 1333-1336, 1982.

Kafemann, H., and U. Motschmann, Studies on nongyrotropic ion distributions in magneto plasmas by $2 \mathrm{D}$ hybrid simulation, Simpo Newsl. 5, 14-19, 1995.

Mauk, B. H., Electromagnetic wave energization of heavy ions by the electric "phase bunching" process, Geophys. Res. Lett., 9, 1163-1166, 1982.

Motschmann, U., and K. H. Glassmeier, Nongyrotropic distribution of pickup ions at comet $\mathrm{P} / \mathrm{Grigg}$-Skjellerup: A possible source of wave activity, J. Geophys. Res., 98, 20977-20983, 1993.

Motschmann, U., K. Sauer, T. Roatsch, and J. F. McKenzie, Subcritical multiple-ion shocks, J. Geophys. Res., 96A, 1384113848, 1991

Neubauer, F. M., K. H. Glassmeier, A. J. Coates, and A. D. Johnston, Low-frequency electromagnetic plasma waves at comet P/Grigg-Skjellerup: analysis and interpretation, J. Geophys. Res., 98, 20937-20953, 1993.

Scholer, M., M. Fujimoto, and H. Kucharek, Two-dimensional simulations of supercritical quasi-parallel shocks: Upstream waves, downstream waves, and shock re-formation, J. Geophys. Res., 98, 18971-18984, 1993.

Sckopke, N., G. Paschmann, A. L. Brinca, C. W. Carlson, and H. Lühr, Ion thermalisation in quasi-perpendicular shocks involving reflected ions, J. Geophys. Res., 95, 6337-6352, 1990.

Sudan, R. N., Growing waves in a nongyrotropic plasma, Phys. Fluids, 8, 1915-1918, 1965.

Thomsen, M. F., J. T. Gosling, S. J. Bame, and C. T. Russell, Gyrating ions and large-amplitude monochromatic MHD waves upstream of the earth's bow shock, J. Geophys. Res., 90, 267-273, 1985. 